\title{
SOME THEOREMS ON FOURIER TRANSFORMS AND CONJUGATE TRIGONOMETRIC INTEGRALS $\dagger$
}

\author{
BY \\ R. P. BOAS, JR.
}

1. Introduction. Functions whose Fourier transforms vanish for large values of the argument have been studied by Paley and Wiener. $\neq$ It is natural, then, to ask what properties are possessed by functions whose Fourier transforms vanish over a finite interval. The two classes of functions are evidently closely related, since a function whose Fourier transform vanishes on $(-A, A)$ is the difference of two suitably chosen functions, one of which has its Fourier transform vanishing outside $(-A, A)$. In this paper, however, we obtain, for a function having a Fourier transform vanishing over a finite interval, a criterion which does not seem to be trivially related to known results. It is stated in terms of a transform which is closely related to the Hilbert transform, and which may be written in the simplest (and probably most interesting) case,

$$
f^{*}(x)=\frac{1}{\pi} \int_{0}^{\infty} \frac{f(x+t)-f(x-t)}{t^{2}} \sin t d t .
$$

With this notation, we shall show that a necessary and sufficient condition for a function $f(x)$ belonging to $L^{2}(-\infty, \infty)$ to have a Fourier transform vanishing almost everywhere on $(-1,1)$ is that $f^{* *}(x)=-f(x)$ almost everywhere; $f^{* *}(x)$ is the result of applying the transform (1) to $f^{*}(x)$. The result holds also if $(\sin t) / t$ is replaced in the integrand in (1) by a certain more general function $H(t)$.

As a preliminary result, we discuss the effect of the general transform $f^{*}(x)$ on a trigonometric Stieltjes integral,

$$
f(x)=\int_{-R}^{R} e^{i x t} d \alpha(t)
$$

where $\alpha(t)$ is a normalized $\S$ complex-valued function of bounded variation. It is found that if $\alpha(t)$ is constant on $-1<t<0$ and on $0<t<1$, then $f^{*}(x)$ is equal to the conjugate trigonometric integral,

$\dagger$ Presented to the Society, April 10, 1936; received by the editors, January 10, 1936.

$\ddagger$ R. E. A. C. Paley and N. Wiener, Fourier Transforms in the Complex Domain, American Mathematical Society Colloquium Publications, vol. 19, 1934, pp. 13, 24, 68 ff.

$\S \alpha(t)$ is said to be normalized if $\alpha(t)=\frac{1}{2}[\alpha(t+0)+\alpha(t-0)],-R<t<R$. 


$$
\bar{f}(x)=i \int_{-R}^{R} e^{i x t} \operatorname{sgn} t d \alpha(t)
$$

This includes the case where $f(x)$ is a trigonometric polynomial. $\ddagger$

The relation $\bar{f}(x)=f^{*}(x)$, when valid, proves to be effective for establishing inequalities for $\bar{f}(x)$. For this application we shall need also generalizations of S. Bernstein's classical inequality for the derivative of a trigonometric polynomial;§ these we obtain in $\$ 4$. For the sake of completeness it has seemed desirable to establish stronger results than we actually need, and which generalize to integrals of the form (2) extensions of Bernstein's theorem which have been given for trigonometric polynomials by G. Szegö\| and by A. Zygmund. $\uparrow$

Bernstein's theorem states that if

$$
f(x)=\frac{a_{0}}{2}+\sum_{n=1}^{R}\left(a_{n} \cos n x+b_{n} \sin n x\right)
$$

is a trigonometric polynomial of order $R$ (with real coefficients), and if $|f(x)| \leqq M,-\infty<x<\infty$, then $\left|f^{\prime}(x)\right| \leqq M R,-\infty<x<\infty$. Bernstein has shown $\dagger$ that the same conclusion follows if $f(x)$ is merely an entire function with the property that $\lim \sup _{n \rightarrow \infty}\left|f^{(n)}(x)\right|^{1 / n}=R$; this includes functions $f(x)$ of the form (2), and would be sufficient for our purposes.

When $f(x)$ is a trigonometric polynomial, Szegö's result is that $|f(x)| \leqq M$ implies not only Bernstein's inequality but also

$$
\left|R \sigma(x)+\bar{f}^{\prime}(x) \cos \theta+f^{\prime}(x) \sin \theta\right| \leqq M R
$$

for any real $\theta$, where

$$
\sigma(x)=\frac{a_{0}}{2}+\sum_{n=1}^{R-1}\left(1-\frac{n}{R}\right)\left(a_{n} \cos n x+b_{n} \sin n x\right)
$$

is an arithmetic mean of $f(x)$. An equivalent statement is

$\dagger \operatorname{sgn} t=t /|t|, t \neq 0 ; \operatorname{sgn} 0=0$.

$\ddagger$ Except that our definition of the conjugate yields the negative of the usual conjugate in the case of a trigonometric polynomial; our conjugate is so defined that it will coincide with the Hilbert transform.

$\S \mathrm{I}$ am indebted to Professor D. V. Widder for originally calling my attention to the question of generalizing Bernstein's theorem to trigonometric integrals.

|| G. Szegö, Über einen Satz des Herrn Serge Bernstein, Schriften der Königsberger gelehrten Gesellschaft, vol. 5 (1928), pp. 59-70.

T A. Zygmund, A remark on conjugate series, Proceedings of the London Mathematical Society, (2), vol. 34 (1932), pp. 392-400.

†† S. Bernstein, Leçons sur les Propriétés Extrémales et la Meilleure Approximation des Fonctions Analytiques d'une Variable Réelle, 1926, p. 102. 


$$
R|\sigma(x)|+\left[\left(\bar{f}^{\prime}(x)\right)^{2}+\left(f^{\prime}(x)\right)^{2}\right]^{1 / 2} \leqq M R .
$$

Zygmund showed that if $\phi(u)$ is a non-decreasing convex function of $u(u \geqq 0)$, then

$$
\int_{0}^{2 \pi} \phi\left(\frac{\left|f^{\prime}(x)\right|}{R}\right) d x \leqq \int_{0}^{2 \pi} \phi(|f(x)|) d x
$$

with the same inequality for $\phi\left(\left|\bar{f}^{\prime}(x)\right| / R\right)$.

These are the results of Szegö and Zygmund which we generalize. We show that if $f(x)$ has the form (2), with $\bar{\alpha}(t)=\alpha(-t), \dagger$ and if $|f(x)| \leqq M$, $-\infty<x<\infty$, then (3) holds, where

$$
\sigma(x)=\int_{-R}^{R}\left(1-\frac{|t|}{R}\right) e^{i x t} d \alpha(t)
$$

and that Zygmund's result may be generalized to the inequality

$$
\int_{-\infty}^{\infty} \phi\left(\left|\sigma(x)+\frac{\cos \theta}{R} \bar{f}^{\prime}(x)+\frac{\sin \theta}{R} f^{\prime}(x)\right|\right) d x \leqq \int_{-\infty}^{\infty} \phi(|f(x)|) d x,
$$

where $\phi(u)$ is non-negative, non-decreasing, and convex.

From (3) we may also obtain the generalization to integrals of a result of van der Corput and Schaake, $\ddagger$ namely, that under the same hypotheses on $f(x)$,

$$
\left|f^{\prime}(x)\right| \leqq R\left[M^{2}-(f(x))^{2}\right]^{1 / 2} .
$$

In $\$ 5$ we apply our results to obtain inequalities for conjugate trigonometric integrals. We can generalize to trigonometric integrals a theorem of $\mathrm{M}$. Fekete, $\S$ which states that if $f(x)$ is measurable on $(-\pi, \pi)$ and periodic with the period $2 \pi$; if $|f(x)| \leqq M$; and if $\bar{s}_{n}(x)$ is the sum of the first $n$ terms of the series conjugate to the Fourier series of $f(x)$, then $\left|\bar{s}_{n}(x)\right| \leqq C M \log n$, where $C$ is an absolute constant. We shall show that if $f(x)$ has the form (2), with $\bar{\alpha}(t)=\alpha(-t)$, and $\alpha(t)$ constant on $0<t<1$, then $|f(x)| \leqq M,-\infty<x<\infty$, implies

$\dagger$ Professor Szegö has pointed out to me that this last restriction may be removed by an elementary argument of a familiar type. The same remark applies to the inequality (4). (Added in proof.)

$\ddagger$ J. G. van der Corput and G. Schaake, Ungleichungen für Polynome und trigonometrische Polynome, Compositio Mathematica, vol. 2 (1935), pp. 321-361; p. 337. The inequality is actually implicit in Szegö's inequality (3).

$\S$ M. F.kete, Über einen Satz des Herrn Serge Bernstein, Journal für die reine und angewandte Mathematik, vol. 146 (1916), pp. 88-94; p. 91. Fekete gives no proof; the only proof which I have seen is that given by T. Carleman, Über die Fourierkoefizienten einer stetigen Funktion, Acta Mathematica, vol. 41 (1918), pp. 377-384; p. 383. I am indebted to Professor G. H. Hardy for calling my attention to the problem of bounds for the conjugate of a trigonometric polynomial. 


$$
|\bar{f}(x)|<M\left(A+\frac{2}{\pi} \log R\right),
$$

where $A$ is an absolute constant $<4 / \pi$. The constant $2 / \pi$ multiplying $\log R$ is the best possible constant. Our result is less precise than Fekete's in that it requires a more restrictive hypothesis on $f(x)$; on the other hand, it applies to a wider class of functions, and fixes the best possible value of the constant $C$.

The analogy between the inequality (4) and S. Bernstein's inequality for derivatives suggests that Zygmund's generalization (mentioned above) of Bernstein's theorem to an inequality for a mean value of the derivative should have an analogue for the conjugate integral. We establish such an analogue in Theorem 8 .

2. A preliminary theorem. We consider first trigonometric Stieltjes integrals over a finite range; that is, functions of the form

$$
f(x)=\int_{-R}^{R} e^{i x t} d \alpha(t),
$$

where $\alpha(t)$ is a normalized complex-valued function of the real variable $t$, of bounded variation on $(-R, R)$. There is no loss in generality from assuming $R>1$. Then we write

$$
\begin{aligned}
& f_{1}(x)=\int_{-1}^{1} e^{i x t} d \alpha(t) \\
& f_{2}(x)=\left(\int_{-R}^{-1}+\int_{1}^{R}\right) e^{i x t} d \alpha(t),
\end{aligned}
$$

so that $f(x)=f_{1}(x)+f_{2}(x)$.

We now introduce the notation, for any $f(x)$ defined on $(-\infty, \infty)$,

$$
f^{*}(x)=\frac{1}{\pi} \int_{0}^{\infty} \frac{f(x+t)-f(x-t)}{t} H(t) d t,
$$

for any $x$ for which the integral exists in the sense of $\lim _{\epsilon \rightarrow 0, T \rightarrow \infty} \int_{\epsilon}^{T} . H(t)$ is a function satisfying Conditions A, below.

Let us denote by $\bar{f}(x)$ the Hilbert transform $\dagger$ of $f(x)$,

$$
\bar{f}(x)=\frac{1}{\pi} \int_{0}^{\infty} \frac{f(x+t)-f(x-t)}{t} d t .
$$

† We shall use the bar to denote both the Hilbert transform and the complex conjugate, but no confusion should result. 
It is known that if $f(x)$ belongs to $L^{2}(-\infty, \infty)$, then $\bar{f}(x)$ exists for almost all $x$, and also belongs to $L^{2}(-\infty, \infty)$. $\dagger$

We shall require $H(t)$ to satisfy the following conditions.

Conditions A. $1 . h(s)$ is a continuous function of bounded variation on $-1 \leqq s \leqq 1$, and

$$
H(t)=\int_{-1}^{1} \cos s t d h(s) .
$$

2. For whatever class of functions $f(x)$ is under consideration, $H(t)$ shall be such that the integral (6) converges with respect to the upper limit whenever (7) does so.

The second condition will in particular be satisfied if $H(t)$ has bounded variation on $(T, \infty)$ for some $T$; or if $h(s)$ is the integral of a function $k(s)$ of bounded variation on $(-1,1)$, and the class of functions $f(x)$ is the class of functions bounded as $x \rightarrow \infty$.

We shall establish the following preliminary theorem.

THEOREM 1. If $f(x)$ and $f_{2}(x)$ are defined by (2) and (5), and if $H(t)$ satisfies Conditions A, then $f^{*}(x)$ exists for all $x$, and

$$
f^{*}(x)=H(0) \bar{f}_{2}(x)+i \int_{-1}^{1} e^{i x t}[h(t)-h(-t)] d \alpha(t) .
$$

It will be sufficient to establish the theorem for the special case

$$
f(x)=\int_{-R}^{R} \sin x t d \beta(t),
$$

where $\beta(t)$ is a normalized function of bounded variation, and for the special point $x=0$. In this case (8) becomes

$$
f^{*}(0)=H(0) \bar{f}_{2}(0)+\int_{-1}^{1}[h(t)-h(-t)] d \beta(t) .
$$

To see this, $\ddagger$ suppose Theorem 1 established in this special case, and set, with $f(x)$ now defined by $(2)$,

$\dagger$ N. Lusin, Sur la convergence des séries trigonométriques de Fourier, Paris Comptes Rendus, vol. 156 (1913), pp. 1655-1658. For references to other proofs, see A. Zygmund, Trigonometrical Series, 1935, Chapter 7; and G. H. Hardy and J. E. Littlewood, The allied series of a Fourier series, Proceedings of the London Mathematical Society, (2), vol. 24 (1925), pp. 211-246.

¥ This device was suggested by a remark made by L. Fejér and by E. Landau, in connection with S. Bernstein's theorem for derivatives. Fekete, op. cit., pp. 88, 93, attributes the idea to Fejér; Bernstein, Sur une propriété des fonctions entières, Paris Comptes Rendus, vol. 176 (1923), pp. 16031605 , attributes it to Landau. 


$$
\begin{aligned}
g(y) & =\frac{1}{2}[f(x+y)-f(x-y)]=\frac{1}{2} \int_{-R}^{R}\left[e^{i(x+y) t}-e^{i(x-y) t}\right] d \alpha(t), \\
\text { (10) } \quad g(y) & =\int_{-R}^{R} \sin y t d \beta(t), \quad \beta(t)=\int_{-R}^{t} i e^{i x u} d \alpha(u) .
\end{aligned}
$$

Then $g(y)$ has the form (9), and

$$
\begin{aligned}
g^{*}(y)= & \frac{1}{\pi} \int_{0}^{\infty} \frac{g(y+t)-g(y-t)}{t} H(t) d t \\
= & \frac{1}{2 \pi} \int_{0}^{\infty} \frac{f(x+y+t)-f(x+y-t)}{t} H(t) d t \\
& +\frac{1}{2 \pi} \int_{0}^{\infty} \frac{f(x-y+t)-f(x-y-t)}{t} H(t) d t \\
= & \frac{1}{2}\left[f^{*}(x+y)+f^{*}(x-y)\right] .
\end{aligned}
$$

Thus, $g^{*}(0)=f^{*}(x)$. Similarly, $\bar{g}(0)=\bar{f}(x)$. Finally,

$$
\int_{-1}^{1}[h(t)-h(-t)] d \beta(t)=i \int_{-1}^{1} e^{i x t}[h(t)-h(-t)] d \alpha(t),
$$

as we see by referring to (10).

We assume that the relation

$$
\bar{f}(x)=i \int_{-R}^{R} e^{i x t} \operatorname{sgn} t d \alpha(t)
$$

for functions of the form (2), and in particular

$$
\bar{f}(x)=\int_{-R}^{R} \cos x t \operatorname{sgn} t d \beta(t)
$$

for functions of the form (9), is known to hold for all $x$; it is easily established by substituting the explicit expression for $f(x)$ into (7), and changing the order of integration. $\dagger$

We now show that if $f(x)$ is defined by $(9), f^{*}(0)$ exists and satisfies (8).

We have, since $f(x)$ is an odd function,

$$
\begin{aligned}
\bar{f}(0) & =\frac{2}{\pi} \int_{0}^{\infty} \frac{f(t)}{t} d t \\
f^{*}(0) & =\frac{2}{\pi} \int_{0}^{\infty} \frac{f(t) H(t)}{t} d t
\end{aligned}
$$

$\dagger$ This fact is implicit in a number of places; but I do not know where to refer for an explicit statement. 
provided that the integrals exist. The convergence of the first integral is already known; the second integral converges with respect to the upper limit, because of Conditions $\mathrm{A}$, and its convergence at the lower limit follows from the existence of $f^{\prime}(0)$.

Consider now

$$
\begin{aligned}
f^{*}(0) & =\frac{2}{\pi} \int_{0}^{\infty} \frac{f(t)}{t} H(t) d t \\
& =\frac{2}{\pi} \int_{0}^{\infty} \frac{d t}{t} \int_{-R}^{R} \sin t u d \alpha(u) \int_{-1}^{1} \cos s t d h(s) \\
& =\frac{2}{\pi} \int_{-R}^{R} d \alpha(u) \int_{-1}^{1} d h(s) \int_{0}^{\infty} \frac{\sin u t \cos s t}{t} d t
\end{aligned}
$$

provided that the change of the order of integration is justified. Assuming this for the present, we have

$$
\int_{0}^{\infty} \frac{\sin u t \cos s t}{t} d t=\left\{\begin{array}{l}
\frac{\pi}{2} \operatorname{sgn} u,\left|\frac{s}{u}\right|<1 ; \\
\frac{\pi}{4} \operatorname{sgn} u,\left|\frac{s}{u}\right|=1 ; \\
0,\left|\frac{s}{u}\right|>1 ; s=u=0 ;
\end{array}\right.
$$

by a familiar formula. Thus,

$$
\begin{aligned}
f^{*}(0) & =\left(\int_{-R}^{-1}+\int_{1}^{R}\right) \operatorname{sgn} u d \alpha(u) \int_{-1}^{1} d h(s)+\int_{-1}^{1} \operatorname{sgn} u d \alpha(u) \int_{-|u|}^{|u|} d h(s) \\
& =H(0) \bar{f}_{2}(0)+\int_{-1}^{1}[h(u)-h(-u)] d \alpha(u)
\end{aligned}
$$

as desired. It remains to justify the change of the order of integration in (13). We have to show that

$$
\begin{aligned}
\left(\int_{0}^{T}+\int_{T}^{\infty}\right) & \frac{d t}{t} \int_{-R}^{R} \sin u t d \alpha(u) \int_{-1}^{1} \cos s t d h(s) \\
& =\int_{-R}^{R} d \alpha(u) \int_{-1}^{1} d h(s)\left(\int_{0}^{T}+\int_{T}^{\infty}\right) \frac{\sin u t \cos s t}{t} d t .
\end{aligned}
$$

We note first that the improper integrals in (14) are actually convergent. Since the integrals over the finite part of the range are clearly equal, it is only necessary to show that $\lim _{T \rightarrow \infty} J(T)=0$, where 


$$
\begin{aligned}
J(T) & =\int_{T}^{\infty} \frac{f(t) H(t)}{t} d t-\int_{-R}^{R} d \alpha(u) \int_{-1}^{1} d h(s) \int_{T}^{\infty} \frac{\sin u t \cos s t}{t} d t \\
& =J_{1}-J_{2} .
\end{aligned}
$$

We have

$$
\begin{aligned}
2 J_{2}= & \int_{-R}^{R} d \alpha(u) \int_{-1}^{1} d h(s) \int_{T}^{\infty} \frac{\sin (u+s) t}{t} d t \\
& +\int_{-R}^{R} d \alpha(u) \int_{-1}^{1} d h(s) \int_{T}^{\infty} \frac{\sin (u-s) t}{t} d t \\
= & J_{3}+J_{4} .
\end{aligned}
$$

It is a familiar fact in the theory of Fourier series that

$$
\left|\int_{a}^{b} \frac{\sin x t}{t} d t\right| \leqq M
$$

where $M$ is independent of $x, a$, and $b$; and that

$$
\lim _{T \rightarrow+\infty} \int_{T_{x}}^{\infty} \frac{\sin v}{v} d v=0
$$

uniformly for $x \geqq x_{0}>0$.

In $J_{3}$ the integral with respect to $s$ over $(-u-\delta,-u+\delta)$ is $o(1)$ for $\delta \rightarrow 0$, since $h(s)$ is continuous; since the integral with respect to $t$ is uniformly bounded, by (15); and since $\alpha(u)$ has bounded variation. (It is supposed that $h(s)$ is extended outside $(-1,1)$, if necessary, so that it is continuous at \pm 1 , and constant outside $(-1,1)$.) Similarly, in $J_{4}$ the integral with respect to $s$ over $(u-\delta, u+\delta)$ is $o(1)$. Now, given any $\epsilon>0$, we may take $\delta$ so small that these two terms are both less in absolute value than $\epsilon$, and then fix $\delta$. In the remaining part of $J_{3}$, we have $|u+s| \geqq \delta$, and

$$
\int_{T}^{\infty} \frac{\sin (u+s) t}{t} d t= \pm \int_{T|u+s|}^{\infty} \frac{\sin v}{v} d v=o(1) \quad(T \rightarrow \infty)
$$

by (16). Similar reasoning applies to $J_{4}$.

Finally, $J_{1}$ is the remainder of a convergent integral, and hence $J_{1}=o(1)$ as $T \rightarrow \infty$. Thus $\lim _{T \rightarrow \infty} J(T)=0$, and Theorem 1 is fully established.

COROLLARY 1. If $f(x)$ satisfies the hypotheses of Theorem 1 ,

$$
\frac{1}{\pi} \int_{0}^{\infty} \frac{f(x+t)-f(x-t)}{t^{2}} \sin t d t=-2 f_{1}^{\prime}(x)+\bar{f}_{2}(x) .
$$

This is the special case $h(s)=s$. 
CoROllary 2. If $f(x)$ satisfies the hypotheses of Theorem 1 , if $H(0)=1$, and if $\alpha(t) \equiv$ constant, $-1<t<0$, and $0<t<1$, then $f^{*}(x)=\bar{f}(x)$.

This includes the case where $f(x)$ is a trigonometric polynomial,

$$
f(x)=\frac{a_{0}}{2}+\sum_{k=0}^{n}\left(a_{k} \cos k x+b_{k} \sin k x\right) .
$$

Corollary 3. If $f(x)$ satisfies the hypotheses of Theorem 1 , and if $H(0)=0$, then

$$
f^{*}(x)=i \int_{-1}^{1} e^{i x t}[h(t)-h(-t)] d \alpha(t) .
$$

3. Functions whose Fourier transforms vanish on $(-1,1)$. We shall need a result of $\mathrm{E}$. $\mathrm{C}$. Titchmarsh, which we state as a lemma.

Lemma 1. $\dagger$ If $f(x)$ belongs to $L^{2}(-\infty, \infty)$, and $F(x)$ is its Fourier transform, then the Fourier transform of $\bar{f}(x)$ is $E(x)=-i F(x) \operatorname{sgn} x$.

We can now establish our main theorem.

ThEOREM 2. Let $f(x)$ belong to $L^{2}(-\infty, \infty)$; let $F(x)$ be the Fourier transform of $f(x)$. Let $H(t)$ satisfy Conditions $\mathrm{A}$, and the further conditions that $H(0)=1$, and that $h(s)-h(-s)= \pm 1$ almost nowhere. A necessary and suffcient condition that $F(x)=0$, almost everywhere on $(-1,1)$, is that

$$
f^{* *}(x)=-f(x)
$$

almost everywhere.

Here $f^{*}(x)$ is defined by (6), and $f^{* *}(x)$ is related to $f^{*}(x)$ as $f^{*}(x)$ is to $f(x)$. We establish first the necessity of the condition. We notice first that

$$
G(t)=\frac{1}{t}(1-H(t))
$$

is a function of $L^{2}$, and that its Fourier sine transform is

$$
g(t)=\left(\frac{2}{\pi}\right)^{1 / 2} \int_{0}^{\infty} G(u) \sin u t d u= \begin{cases}\left(\frac{\pi}{2}\right)^{1 / 2}(1-h(t)+h(-t)), \\ 0,|t| \geqq 1 . & -1 \leqq t \leqq 1,\end{cases}
$$

$\dagger$ E. C. Titchmarsh, Conjugate trigonometric integrals, Proceedings of the London Mathematical Society, (2), vol. 24 (1925), pp. 109-130; p. 114. 
By Plancherel's theorem, for almost all $x$

$$
f(x)=\frac{1}{(2 \pi)^{1 / 2}} \underset{a \rightarrow \infty}{\lim .} \int_{-a}^{a} e^{-i x u} F(u) d u .
$$

Let now $x$ be given and fixed. Then for almost all $t$, by (19),

$$
\begin{aligned}
k(t) \equiv f(x+t)-f(x-t) & =\frac{-2 i}{(2 \pi)^{1 / 2}} \underset{a \rightarrow \infty}{\operatorname{li} . \mathrm{m} .} \int_{-a}^{a} e^{-i x u} F(u) \sin t u d u \\
& =\left(\frac{2}{\pi}\right)^{1 / 2} \underset{\substack{\operatorname{li} . \mathrm{m} \\
a \rightarrow \infty}}{\int_{0}^{a} \sin t u K(u) d u,}
\end{aligned}
$$

where $K(u)=-i\left[e^{-i x u} F(u)-e^{i x u} F(-u)\right]$, so that $K(u)=0$ almost everywhere on $(0,1)$.

By the Parseval relation for Fourier transforms,

$$
\int_{0}^{\infty} k(y) G(y) d y=\int_{0}^{\infty} K(y) g(y) d y=0,
$$

since $g(y) \equiv 0$ on $(1, \infty)$, and $K(y)=0$ almost everywhere on $(0,1)$. Referring to (17) and (20), we see that (21) is equivalent to $\bar{f}(x)=f^{*}(x)$ whenever either of these functions exists; and $\bar{f}(x)$ exists almost everywhere.

Now $\bar{f}(x)$ belongs to $L^{2}(-\infty, \infty)$; let $E(x)$ be the Fourier transform of $\bar{f}(x)$. By Lemma $1, E(x)=-i F(x) \operatorname{sgn} x$, so that $E(x)=0$ almost everywhere on $(-1,1)$. We may now apply our previous reasoning, with only formal modifications, to $\bar{f}(x)$, and find

$$
-f(x)=\bar{f}(x)=f^{* *}(x) .
$$

The necessity of the condition is thus established.

Now let $f(x)$ belonging to $L^{2}(-\infty, \infty)$ have $f^{* *}(x)=-f(x)$. Set

$$
k(x)=f(x)-\frac{1}{(2 \pi)^{1 / 2}} \int_{-1}^{1} F(t) e^{-i x t} d t,
$$

where $F(x)$ is the Fourier transform of $f(x)$. It is clear that $k(x)$ belongs to $L^{2}(-\infty, \infty)$; hence $k(x)$ has a Fourier transform $K(x)$. It is clear that $K(x)=0$ almost everywhere on $(-1,1)$. Hence, by what has already been proved, $k^{* *}(x)=-k(x)$. But, by Theorem 1 and our hypothesis,

$$
k^{* *}(x)=-f(x)+\frac{1}{(2 \pi)^{1 / 2}} \int_{-1}^{1} F(t)[h(t)-h(-t)]^{2} e^{-i x t} d t .
$$

Hence, 


$$
\int_{-1}^{1} F(t)\left(1-[h(t)-h(-t)]^{2}\right) e^{-i x t} d t=0
$$

for almost all $x$. This is possible only if

$$
F(t)\left(1-[h(t)-h(-t)]^{2}\right)=0
$$

almost everywhere on $(-1,1)$. And since almost everywhere $h(t)-h(-t)$ $\neq \pm 1$, we have finally $F(t)=0$, almost everywhere on $(-1,1)$. This completes the proof of the theorem.

In Theorem 2, we assumed that $H(0)=1$. This assumption was made to ensure that $H(0) \neq 0$, so that it was possible to divide by it. It is natural to ask what happens to Theorem 2 if we allow $H(0)$ to vanish. We establish the following theorem.

TheOREM 3. Let $f(x)$ belong to $L^{2}(-\infty, \infty)$, and let $F(x)$ be the Fourier transform of $f(x)$. Let $H(t)$ satisfy Conditions $\mathrm{A}$; let $H(0)=0$, and let $h(s)=h(-s)$ almost nowhere. A necessary and sufficient condition that $F(x)=0$ almost everywhere on $(-1,1)$ is that $f^{*}(x)=0$ almost everywhere.

The proof is much the same as that of Theorem 3, but simplified because Lemma 1 is no longer needed.

To establish the necessity of the condition, we set now $G(t)=H(t) / t$. Since $H(0)=0$, we find that the Fourier sine transform of $G(t)$ is

$$
g(t)=\left(\frac{2}{\pi}\right)^{1 / 2} \int_{0}^{\infty} G(u) \sin u t d u=\left\{\begin{array}{l}
\left(\frac{\pi}{2}\right)^{1 / 2}[h(t)-h(-t)], \\
0,|t| \geqq 1 .
\end{array}\right.
$$

The relation (20) holds as before, and (21) holds also, if $g(t)$ is defined by (23). But now

almost everywhere.

$$
\frac{1}{\pi} \int_{0}^{\infty} k(y) G(y) d y=f^{*}(x)=0
$$

On the other hand, if $f^{*}(x)=0$ almost everywhere, we define $k(x)$ by (22), and $K(x)$ as before. Since $K(x)$ clearly vanishes almost everywhere on $(-1,1)$, the first part of the proof gives

$$
k^{*}(x)=\frac{-i}{(2 \pi)^{1 / 2}} \int_{-1}^{1} F(t)[h(t)-h(-t)] e^{-i x t} d t=0
$$

for almost all $x$. This can happen, since $h(t)-h(-t)$ is almost nowhere zero, only if $F(t)=0$ almost everywhere on $(-1,1)$. 
4. Inequalities for the derivative of a trigonometric integral. The generalizations of S. Bernstein's theorem, indicated in the introduction, may be conveniently formulated as follows.

THEOREM 4. Let $f(z)$ be a Laplace integral of the form

$$
f(z)=\int_{0}^{R} e^{-z t} d \alpha(t) \quad(0<R<\infty ; z=x+i y),
$$

where $\alpha(t)$ is a normalized complex-valued function of bounded variation. Let $|\Re[f(i y)]| \leqq M,-\infty<y<\infty$. Let $\theta$ be any real number. Then

$$
\left|\Re\left[R \sigma(i y)+e^{i \theta} f^{\prime}(i y)\right]\right| \leqq M R \quad(-\infty<y<\infty),
$$

where

$$
\sigma(z)=\frac{1}{R} \int_{0}^{R}(R-t) e^{-z t} d \alpha(t)
$$

is an "arithmetic mean" of $f(z)$.

Since (25) holds for all real $\theta$, it yields as an equivalent statement

$$
R|\sigma(i y)|+\left|f^{\prime}(i y)\right| \leqq M R \quad(-\infty<y<\infty),
$$

and as somewhat weaker consequences

$$
\begin{gathered}
\Im\left[f^{\prime}(i y)\right] \leqq R\left[M^{2}-(\Re[f(i y)])^{2}\right]^{1 / 2} \quad(-\infty<y<\infty), \\
\left(\Re\left[R f(i y)-f^{\prime}(i y)\right]\right)^{2}+4\left(\Im\left[f^{\prime}(i y)\right]\right)^{2} \leqq 4 R^{2} M^{2} .
\end{gathered}
$$

Relation (26) follows simply from (25) by use of the formula $R \sigma(z)=R f(z)$ $+f^{\prime}(z)$.

Theorem 5. Let $f(z)$ have the form (24). Let $\phi(u)$ be a non-negative, nondecreasing convex function of $u(u \geqq 0)$. Let $E$ denote $(-\infty, \infty)$ in general, and $(0,2 \pi)$ if $f(i y)$ has the period $2 \pi$ (that is, if $f(z)$ reduces to a polynomial $g(w)$ in $\left.w=e^{-z}\right)$. Then for any real $\theta$

$$
\int_{E} \phi\left(\left|\Re\left[\sigma(i y)+\frac{e^{i \theta}}{R} f^{\prime}(i y)\right]\right|\right) d y \leqq \int_{E} \phi(|\Re[f(i y)]|) d y,
$$

where the integral on the right may be infinite.

Similar integral inequalities corresponding to (26) and (27) may be easily formulated. When $E=(0,2 \pi)$, it is not necessary to require $\phi(u) \geqq 0$.

The results of Theorems 4 and 5 take on a more familiar form if we notice that if $f(z)$ has the form (24), $F(y)=\Re[f(i y)]$ has the form (2) (at least if $\Im(\alpha(t))$ is continuous at $t=0$ ), and that then $\bar{F}(y)=-\Im[f(i y)]$, $F^{\prime}(y)=-\Im\left[f^{\prime}(i y)\right], \bar{F}^{\prime}(y)=-\Re\left[f^{\prime}(i y)\right]$; 


$$
\begin{gathered}
-\Re\left[e^{i \theta} f^{\prime}(i y)\right]=\bar{F}^{\prime}(y) \cos \theta-F^{\prime}(y) \sin \theta, \\
\left(\Re\left[R f(i y)-f^{\prime}(i y)\right]\right)^{2}+4\left(\Im\left[f^{\prime}(i y)\right]\right)^{2}=\left(R F(y)+\bar{F}^{\prime}(y)\right)^{2}+4\left(F^{\prime}(y)\right)^{2} .
\end{gathered}
$$

This allows us to put the results of Theorems 4 and 5 into the form in which they were stated in the introduction. Various other formulations are also possible.

If $f(z)$ is a polynomial in $e^{-z}$, of degree $R,(25)$ contains for $\theta=0$ the original theorem of S. Bernstein, and for general $\theta$ reduces to Szegö's $\dagger$ theorem; (26) becomes a theorem of van der Corput and Schaake, $\dagger$ a consequence of Szegö's theorem; and if $\theta=0$ or $\pi / 2,(28)$ contains two theorems of Zygmund. $\dagger$ If in (24) $\alpha(t)$ is a step-function (with a finite or infinite number of jumps), (25) reduces essentially to a theorem of $M$. Fekete.

We establish Theorems 4 and 5 together, using a modification of the method of F. Riesz.§

We begin with $f(z)$ of the form (24) with $\alpha(t)$ continuous at $t=R$. It is convenient to require that $\alpha(0)=0$; this additional normalization has no effect on $f(z)$. We have, by a familiar inversion formula, $\|$

$$
\beta(t) \equiv \frac{1}{2 \pi i} \int_{-i \infty}^{i \infty} \frac{f(s)}{s} e^{s t} d s= \begin{cases}\alpha(t), & 0<t \leqq R \\ \alpha(R), & t \geqq R ; \\ 0, & t<0 ; \\ \frac{1}{2} \alpha(0+), & t=0 ;\end{cases}
$$

the integral being taken in the Cauchy sense at $s=0$ and $s=\infty$. Then,

$$
\begin{aligned}
-f^{\prime}(0) & =\int_{0}^{R} t d \alpha(t)=R \alpha(R)-\int_{0}^{R} \alpha(t) d t \\
& =R \alpha(R)-\frac{1}{2 \pi i} \int_{0}^{R} d t \int_{-i \infty}^{i \infty} \frac{f(s)}{s} e^{s t} d s \\
& =R \alpha(R)-\frac{1}{2 \pi i} \int_{0}^{R} d t \int_{-\infty}^{\infty} \frac{f(i y)}{y} e^{i y t} d y \\
-f^{\prime}(0) & =R \alpha(R)+\frac{1}{2 \pi} \int_{-\infty}^{\infty} \frac{f(i y)}{y^{2}}\left(e^{i y R}-1\right) d y .
\end{aligned}
$$

$\dagger$ For references, see footnotes on pp. 288, 289.

$\ddagger$ According to Szegö, loc. cit. See also G. Szegö, Zur Theorie der fastperiodischen Funktionen; Mathematische Annalen, vol. 96 (1927), pp. 378-382; and the theorem of S. Bernstein referred to in Theorem 6 .

§ F. Riesz, Sur les polynômes trigonométriques, Paris Comptes Rendus, vol. 158 (1914), pp. 16571661. Similar modifications of the same method have been used by Szegö and by Zygmund.

\| See, e.g., D. V. Widder, A generalization of Dirichlet's series and of Laplace's integrals by means of a Stieltjes integral, these Transactions, vol. 31 (1929), pp. 694-743; p. 708. The result given there needs slight modifications to cover the case in hand. 
The change of order of integration is justified without difficulty. We have also, in the same way,

$$
\begin{aligned}
0 & =\int_{0}^{R} t d \beta(2 R-t) \\
& =R \alpha(R)+\frac{1}{2 \pi} \int_{-\infty}^{\infty} \frac{f(i y)}{y^{2}} e^{2 i R y}\left(1-e^{-i R y}\right) d y \\
0 & =\int_{0}^{R} t d \beta(-t) \\
& =-\frac{1}{2 \pi i} \int_{0}^{R} d t \int_{-i \infty}^{i \infty} \frac{f(s)}{s} e^{-s t} d s
\end{aligned}
$$

and hence,

$$
0=-\frac{1}{2 \pi} \int_{-\infty}^{\infty} \frac{\overline{f(i y)}}{y^{2}}\left(e^{i R y}-1\right) d y,
$$

where $\overline{f(s)}$ is the complex conjugate of $f(s)$. Similarly,

$$
0=\int_{0}^{R} t d \beta(t-2 R)=-\frac{1}{2 \pi} \int_{-\infty}^{\infty} \frac{\overline{f(i y)}}{y^{2}} e^{2 i R y}\left(1-e^{-i R y}\right) d y .
$$

We subtract (30) from (31) and (32) from (33). This gives

$$
\begin{aligned}
f^{\prime}(0) & =\frac{1}{2 \pi} \int_{-\infty}^{\infty} \frac{f(i y)}{y^{2}}\left(e^{i R y}-1\right)^{2} d y, \\
0 & =\frac{1}{2 \pi} \int_{-\infty}^{\infty} \frac{\overline{f(i y)}}{y^{2}}\left(e^{i R y}-1\right)^{2} d y ;
\end{aligned}
$$

and hence, on adding the last two relations,

$$
e^{i \theta} f^{\prime}(0)=\frac{2}{\pi} \int_{-\infty}^{\infty} \Re[f(i y)] e^{i R y+i \theta} \frac{1-\cos R y}{y^{2}} d y .
$$

We take the conjugate of both sides of (34), and add the resulting relation to (34), obtaining

$$
\Re\left[e^{i \theta} f^{\prime}(0)\right]=\frac{2}{\pi} \int_{-\infty}^{\infty} \Re[f(i y)] \cos (\theta+R y) \frac{1-\cos R y}{y^{2}} d y .
$$

In (35) we may replace $\cos (\theta+R y)$ by $g(\theta+R y)$, where $g(u)$ is any integrable function with the period $2 \pi$, having a Fourier series of the form 


$$
g(u) \sim \cos u+\sum_{n=2}^{\infty} a_{n} \cos n u .
$$

For, reasoning similar to that used in deriving (35) shows that

$$
\int_{-\infty}^{\infty} \Re[f(i y)] e^{i k R y} \frac{1-\cos R y}{y^{2}} d y=0 \quad(k= \pm 2, \pm 3, \cdots),
$$

and it is permissible to substitute the Fourier series of $g(u)$ in the integral and integrate term by term. $\dagger$

Now, for any $y_{0}, E(z)=f\left(i y_{0}+z\right)$ has the form (24), and $E^{\prime}(0)=f^{\prime}\left(i y_{0}\right)$, $E(i y)=f\left(i y_{0}+i y\right)$. Hence we have, introducing $g(\theta+R y)$ in (35),

$$
\Re\left[e^{i \theta} f^{\prime}\left(i y_{0}\right)\right]=\frac{2}{\pi} \int_{-\infty}^{\infty} \Re\left[f\left(i y+i y_{0}\right)\right] g(\theta+R y) \frac{1-\cos R y}{y^{2}} d y .
$$

Now, we have

$$
\sigma(i y)=\frac{1}{R} \int_{0}^{R}(R-t) e^{-i y t} d \alpha(t),
$$

and by reasoning similar to that used in obtaining (37), we find

$$
\Re\left[\sigma\left(i y_{0}\right)\right]=\frac{1}{\pi R} \int_{-\infty}^{\infty} \Re\left[f\left(i y+i y_{0}\right)\right] \frac{1-\cos R y}{y^{2}} d y .
$$

Combining this with (37), we obtain

$$
\begin{aligned}
G\left(y_{0}\right) & \equiv\left|\Re\left[R \sigma\left(i y_{0}\right)+e^{i \theta} f^{\prime}\left(i y_{0}\right)\right]\right| . \\
& \leqq \frac{1}{\pi} \int_{-\infty}^{\infty}\left|\Re\left[f\left(i y+i y_{0}\right)\right]\right| \cdot|1+2 g(\theta+R y)| \frac{1-\cos R y}{y^{2}} d y .
\end{aligned}
$$

We now specialize $g(u)$, taking a function nearly the same as that used by Szegö. Set

$$
\begin{aligned}
1+2 g(u)=\frac{1}{2 r}\left\{\frac{(1+r)^{2}(1-r)}{1-2 r \cos u+r^{2}}-\frac{(1-r)^{2}(1+r)}{1+2 r \cos u+r^{2}}\right\} \equiv & h(u) \\
& (0<r<1) .
\end{aligned}
$$

This is readily seen to have the desired form, since

$$
\frac{1-r^{2}}{1-2 r \cos u+r^{2}}=1+2 \sum_{n=1}^{\infty} r^{n} \cos n u \quad(-1<r<1)
$$

† See, e.g., E. W. Hobson, The Theory of Functions of a Real Variable and the Theory of Fourier's Series, vol. 2, 1926, p. 583. 
Then,

$$
|1+2 g(u)| \leqq k(u)=\frac{1}{2 r}\left\{\frac{(1+r)^{2}(1-r)}{1-2 r \cos u+r^{2}}+\frac{(1-r)^{2}(1+r)}{1+2 r \cos u+r^{2}}\right\} .
$$

The function $k(u)$ has a Fourier series of the form

It is easily verified that

$$
k(u)=\frac{1}{r}+\sum_{n=1}^{\infty} c_{n} \cos n u .
$$

so that

$$
\int_{-\infty}^{\infty} \cos n(\theta+R y) \frac{1-\cos R y}{y^{2}} d y=0 \quad(n=1,2, \cdots),
$$

$$
\begin{aligned}
\frac{1}{\pi} \int_{-\infty}^{\infty}|h(\theta+R y)| & \frac{1-\cos R y}{y^{2}} d y \\
& \leqq \frac{1}{\pi} \int_{-\infty}^{\infty} k(\theta+R y) \frac{1-\cos R y}{y^{2}} d y=\frac{R}{r} .
\end{aligned}
$$

We apply this result to (39). We have in the first place, since by hypothesis $\left|\Re\left[f\left(i y+i y_{0}\right)\right]\right| \leqq M$,

$$
G\left(y_{0}\right) \leqq \frac{M R}{r}
$$

and, letting $r \rightarrow 1$,

$$
G\left(y_{0}\right) \leqq R M .
$$

Referring to (41), we obtain (25) of Theorem 4, established now whenever $\alpha(t)$ is continuous at $t=R$. If $\alpha(t)$ is discontinuous at $t=R$, take $R^{\prime}>R$, and write

$$
f(z)=\int_{0}^{R^{\prime}} e^{-z t} d \alpha_{1}(t), \quad \alpha_{1}(t)= \begin{cases}\alpha(t), & t \leqq R \\ \alpha(R), & t \geqq R .\end{cases}
$$

Then $\alpha_{1}(t)$ is continuous at $t=R^{\prime}$, and we deduce

$$
G\left(y_{0}\right) \leqq R^{\prime} M \text {. }
$$

Letting $R^{\prime} \rightarrow R$, we have Theorem 4 fully established.

To obtain Theorem 5 , we introduce the function $k(\theta+R y)$ in (39), apply "Jensen's inequality" for integrals $\nmid$ to the right-hand side, and integrate over $E$. Then, 


$$
\begin{aligned}
\int_{E} \phi\left(\frac{r}{R} G\left(y_{0}\right)\right) d y_{0} & \\
& \leqq \frac{r}{R \pi} \int_{-\infty}^{\infty} k(\theta+R y) \frac{1-\cos R y}{y^{2}} d y \int_{E} \phi\left(\left|\Re\left[f\left(i y+i y_{0}\right)\right]\right|\right) d y_{0} .
\end{aligned}
$$

In the integral over $E$ on the right, apply the periodicity of $f(i y)$ if $E=(0,2 \pi)$, and make an obvious change of variable if $E=(-\infty, \infty)$; then

$$
\int_{E} \phi\left(\frac{r}{R} G\left(y_{0}\right)\right) d y_{0} \leqq \int_{E} \phi\left(\left|\Re\left[f\left(i y_{0}\right)\right]\right|\right) d y_{0} .
$$

Finally, let $r \rightarrow 1$ in (42). We may take the limit under the integral sign, and we obtain the conclusions of Theorem 5 , in the case where $\alpha(t)$ is continuous at $t=R$. This condition may be removed as it was in Theorem 4 .

There is also a generalization of Bernstein's theorem along quite different lines, given by Bernstein himself. Zygmund's generalization of the original theorem suggests the following further generalization.

THEOREM 6. Let $F(z)$ be an entire function such that

$$
\limsup _{n \rightarrow \infty}\left|F^{(n)}(z)\right|^{1 / n}=r>0
$$

for some given $z$ (hence for every $z$ ). Let $\phi(u)$ have the same properties as in Theorem 5 ; let $E$ denote $(0, p)$ if $F(x)$ is a periodic function with period $p$, and $(-\infty, \infty)$ otherwise. Then

$$
\int_{E} \phi\left(\frac{\left|F^{\prime}(x)\right|}{r}\right) d x \leqq \int_{E} \phi(|F(x)|) d x,
$$

where the integral on the right may be infinite.

The theorem of S. Bernstein $\dagger$ states that if $F(z)$ satisfies the hypotheses of Theorem 6, and if $|F(x)| \leqq M,-\infty<x<\infty$, then $\left|F^{\prime}(x)\right| \leqq M r$, $-\infty<x<\infty$. The proof of this theorem given by Pólya and Szegö $\ddagger$ may easily be modified to give a proof of Theorem 6. A function $f(x)$ of the form (2) is a function of the class considered here, with $r=R$.

5. Inequalities for the conjugate of a trigonometric integral. We now apply Theorem 1 , and the results of $\$ 4$, to obtain the generalized theorem of Fekete, mentioned in the introduction.

† S. Bernstein, loc. cit.

‡ G. Pólya and G. Szegö, Aufgaben und Lehrsätze aus der Analysis, 1925, vol. 2, section IV, problem 201, pp. 35, 219. 
TheOREM 7. If $f(x)$ is a trigonometric Stieltjes integral of the special form

$$
f(x)=c+\left(\int_{-R}^{-r}+\int_{r}^{R}\right) e^{i x t} d \alpha(t) \quad(0<r<R),
$$

where $\alpha(t)$ is a normalized, complex-valued function of bounded variation with $\bar{\alpha}(t)=\alpha(-t) ;$ and if $|f(x)| \leqq M,-\infty<x<\infty$, then

$$
|\bar{f}(x)| \leqq\left(A+\frac{2}{\pi} \log \frac{R}{r}\right) M,
$$

where $A$ is an absolute constant $(<4 / \pi)$, independent of $R, r$, and $f(x)$.

$\bar{f}(x)$ is defined by (11) or (7), which are here equivalent. The factor $2 / \pi$ is the best possible in a certain sense to be specified later.

If $\alpha(t)$ is a step-function with jumps only at integral points, $f(x)$ is a trigonometric polynomial, and this theorem, as we stated in the introduction, becomes in one respect more precise, in another respect less precise, than a theorem of Fekete. $\dagger$

We establish Theorem 7 for $r=1$; the general case follows by a change of variable.

By Corollaries 1 and 2 of Theorem 1,

$$
\begin{aligned}
\bar{f}(x) & =\frac{1}{\pi} \int_{0}^{\infty} \frac{f(x+t)-f(x-t)}{t^{2}} \sin t d t \\
\pi|\bar{f}(x)| & \leqq\left|\int_{0}^{1 / R} \frac{f(x+t)-f(x-t)}{t^{2}} \sin t d t\right|+\left|\int_{1 / R}^{i}\right|+\left|\int_{1}^{\infty}\right| .
\end{aligned}
$$

Applying Theorem 4, and the elementary fact that $[f(x+t)-f(x)] / t$ and $f^{\prime}(x)$ have the same upper and lower bounds,

$$
\left|\int_{0}^{1 / R} \frac{f(x+t)-f(x-t)}{t^{2}} \sin t d t\right| \leqq 2 M,
$$

since $|(\sin t) / t| \leqq 1$. Then,

$$
\begin{aligned}
& \left|\int_{1 / R}^{1} \frac{f(x+t)-f(x-t)}{t^{2}} \sin t d t\right| \leqq 2 M \int_{1 / R}^{1} \frac{d t}{t}=2 M \log R, \\
& \left|\int_{1}^{\infty} \frac{f(x+t)-f(x-t)}{t^{2}} \sin t d t\right| \leqq 2 M \int_{1}^{\infty} \frac{|\sin t|}{t^{2}} d t
\end{aligned}
$$

$†$ M. Fekete, loc. cit. 


$$
\begin{aligned}
& <2 M \int_{1}^{\infty} \frac{d t}{t^{2}}=2 M, \\
|\bar{f}(x)| & <\frac{2}{\pi} M(2+\log R) .
\end{aligned}
$$

The constant $2 / \pi$ multiplying $\log (R / r)$ is the best possible constant in the sense that for no constant $B<2 / \pi$ is it true that

$$
\limsup _{R \rightarrow \infty} \frac{\max |\bar{f}(x)|}{\log \frac{R}{r}} \leqq B M
$$

for all $f(x)$ of the form (44), satisfying $|f(x)| \leqq M$. This may be seen by taking for $f(x)$ first order arithmetic means of the partial sums of the series $\sum_{1}^{\infty}(1 / n) \sin n x$.

The value $4 / \pi$ is certainly not the best possible value of the constant $A$; it is easy to improve it slightly by a more accurate estimate of $\int_{1}^{\infty}|\sin t| t^{-2} d t$.

It is clear that if $r=0$, we can say nothing about the order of magnitude of $\bar{f}(x)$. A simple example is furnished by

$$
f(x)=\int_{0}^{1} t^{\delta-1} \sin x t d t \quad\left(0<\delta<\frac{1}{2}\right) .
$$

It is perhaps worth while to remark that since $\bar{f}(x)$ is independent of the constant $c$ in (44), we may make Theorem 7 somewhat sharper by replacing in (45) $M$ by $\frac{1}{2}(k+K)$, where $-k \leqq f(x) \leqq K$.

We turn now to an inequality for a mean value of the conjugate of a trigonometric integral. Applying Theorem 5 (or Theorem 6), we shall prove the following theorem.

THEOREM 8. Let $f(x)$ be (A) a trigonometric polynomial of order $R$, or (B) a trigonometric integral of the form

$$
f(x)=c+\left(\int_{-R}^{-1}+\int_{1}^{R}\right) e^{i x t} d \alpha(t) \quad(R>1),
$$

where $\alpha(t)$ has the same properties as in Theorem 7. Let $\phi(u)$ be a non-negative, non-decreasing convex function of $u(u \geqq 0)$. Let $E$ denote $(0,2 \pi)$ in case (A), and $(-\infty, \infty)$ in case $(\mathrm{B})$. Then

$$
\int_{E} \phi\left(\frac{\pi|\bar{f}(x)|}{4 A(R)}\right) d x \leqq \frac{1}{2} \int_{E}[\phi(2|f(x)|)+\phi(|f(x)|)] d x,
$$

where 


$$
A(R)=\int_{1 / R}^{\infty} \frac{|\sin t|}{t^{2}} d t
$$

and the integral in the right-hand member of (47) may be infinite.

If $R \geqq 2$, we may replace the right-hand side of (47) by

$$
\int_{E} \phi(|f(x)|) d x .
$$

We have $(2 / \pi) \log R+\frac{1}{2}<A(R)<\log R+1$; this evaluation of $A(R)$ brings out the analogy between (47) and (43).

For the case $\phi(y)=y^{r}, r>1$, a stronger result than case (A) of Theorem 8 is known. M. Riesz $\nmid$ has shown that for any $f(x)$ of $L^{r}(0,2 \pi), r>1$ (and hence for $f(x)$ a trigonometric polynomial), there is a constant $A_{r}$ depending only on $r$ such that

$$
\int_{0}^{2 \pi}|\bar{f}(x)|^{r} d x \leqq A_{r}^{r} \int_{0}^{2 \pi}|f(x)|^{r} d x .
$$

On the other hand, our result is still available when $\phi(y)=y$, while the result of Riesz breaks down for $r=1$.

We require an additional lemma, which is a generalization of the fact that the derivative and the difference quotient of a continuous function have the same upper and lower bounds in an interval.

Lemma 3. Let $f(x)$ be absolutely continuous in $a \leqq x \leqq b$. Let $\phi(y)$ be nonnegative, non-decreasing, and convex for $y \geqq 0$. Then

$$
\int_{a}^{b} \phi\left(\left|f^{\prime}(x)\right|\right) d x=M
$$

implies

$$
\int_{c}^{d} \phi\left(\frac{|f(x+t)-f(x-t)|}{2 t}\right) d x \leqq M,
$$

provided that $0 \leqq t<\frac{1}{2}(b-a), c-a>t, b-d>t$.

For the proof we have

$$
J \equiv \int_{c}^{d} \phi\left(\frac{1}{2 t}|f(x+t)-f(x-t)|\right) d x=\int_{c}^{d} \phi\left(\left|\int_{x-t}^{x+t} \frac{f^{\prime}(u)}{2 t} d u\right|\right) d x
$$

† See A. Zygmund, Trigonometrical Series, 1935, p. 147. E. C. Titchmarsh has given a similar result when $\phi(y)$ involves also logarithmic factors: $A$ theorem on conjugate functions, Journal of the London Mathematical Society, vol. 5 (1930), pp. 88-91. 


$$
\leqq \frac{1}{2 t} \int_{c}^{d} d x \int_{x-t}^{x+t} \phi\left(\left|f^{\prime}(u)\right|\right) d u,
$$

by "Jensen's inequality." Then, integrating in the reverse order,

$$
\begin{aligned}
J \leqq & \frac{1}{2 t} \int_{c+t}^{d-t} \phi\left(\left|f^{\prime}(u)\right|\right) d u \int_{u-t}^{u+t} d x+\frac{1}{2 t} \int_{c-t}^{c+t} \phi\left(\left|f^{\prime}(u)\right|\right) d u \int_{c}^{u+t} d x \\
& +\frac{1}{2 t} \int_{d-t}^{d+t} \phi\left(\left|f^{\prime}(u)\right|\right) d u \int_{u-t}^{d} d x \\
\leqq & \int_{c-t}^{d+t} \phi\left(\left|f^{\prime}(u)\right|\right) d u \leqq M .
\end{aligned}
$$

Corollary 1. Lemma 3 remains valid if $a$ or $b$ is infinite, or if both are, with any finite $t \geqq 0$.

COROLlaRy 2. If $f(x)$ has the period $2 \pi$, the conclusion of Lemma 3 holds with $a=c=0, b=d=2 \pi, 0 \leqq t \leqq 2 \pi$.

This follows from a more precise evaluation of the integrals in the last step of the proof.

We are now in a position to establish Theorem 8 . We have

$$
\begin{aligned}
\pi|\bar{f}(x)| \leqq & \int_{0}^{1 / R} \frac{|f(x+t)-f(x-t)|}{t} d t \\
& +\int_{1 / R}^{\infty} \frac{|f(x+t)-f(x-t)|}{t^{2}}|\sin t| d t,
\end{aligned}
$$

as in the proof of Theorem 7. Applying the convexity of $\phi(y)$,

$$
\begin{aligned}
2 \phi\left(\frac{\pi|\bar{f}(x)|}{4 A(R)}\right) \leqq \phi( & \left.\frac{1}{A(R)} \int_{0}^{1 / R} \frac{|f(x+t)-f(x-t)|}{2 t} d t\right) \\
& +\phi\left(\frac{1}{A(R)} \int_{1 / R}^{\infty} \frac{|f(x+t)-f(x-t)|}{2 t^{2}}|\sin t| d t\right) .
\end{aligned}
$$

To the two terms on the right we apply Jensen's inequality. This gives

$$
\begin{aligned}
2 \phi\left(\frac{\pi|\bar{f}(x)|}{4 A(R)}\right) \leqq R & \int_{0}^{1 / R} \phi\left(\frac{|f(x+t)-f(x-t)|}{2 R t A(R)}\right) d t \\
& +\frac{1}{A(R)} \int_{1 / R}^{\infty} \phi\left(\frac{|f(x+t)-f(x-t)|}{2}\right) \frac{|\sin t|}{t^{2}} d t .
\end{aligned}
$$

Now, $\phi(y)$ is an increasing function. In the first term on the right, we use the fact that $1 / A(R)<2$; in the second term, we use the inequality 
$|f(x+t)-f(x-t)| \leqq|f(x+t)|+|f(x-t)|$, and apply the convexity of $\phi(y)$. This gives us

$$
\begin{aligned}
2 \phi\left(\frac{\pi|\bar{f}(x)|}{4 A(R)}\right) & \leqq R \int_{0}^{1 / R} \phi\left(\frac{|f(x+t)-f(x-t)|}{R t}\right) d t \\
& +\frac{1}{2 A(R)} \int_{1 / R}^{\infty}[\phi(|f(x+t)|)+\phi(|f(x-t)|)] \frac{|\sin t|}{t^{2}} d t
\end{aligned}
$$

Now we integrate both sides of (50) over $E$, and change the order of integration, obtaining

$$
\begin{aligned}
2 \int_{E} \phi & \left(\frac{\pi|\bar{f}(x)|}{4 A(R)}\right) d x \leqq R \int_{0}^{1 / R} d t \int_{E} \phi\left(\frac{|f(x+t)-f(x-t)|}{R t}\right) d x \\
& +\frac{1}{2 A(R)} \int_{1 / R}^{\infty} \frac{|\sin t|}{t^{2}} d t \int_{E}[\phi(|f(x+t)|)+\phi(|f(x-t)|)] d x
\end{aligned}
$$

Apply the appropriate corollary of Lemma 3 to the first term on the right of (51); in the second term, apply the periodicity of $f(x)$ in case (A), and make an obvious change of variable in case $(B)$; then,

$$
\begin{aligned}
2 \int_{E} \phi\left(\frac{\pi|\bar{f}(x)|}{4 A(R)}\right) d x \leqq & R \int_{0}^{1 / R} d t \int_{E} \phi\left(\frac{2\left|f^{\prime}(x)\right|}{R}\right) d x \\
& +\frac{1}{A(R)} \int_{1 / R}^{\infty} \frac{|\sin t|}{t^{2}} d t \int_{E} \phi(|f(x)|) d x \\
\leqq & \int_{E} \phi(2|f(x)|) d x+\int_{E} \phi(|f(x)|) d x
\end{aligned}
$$

by Theorem 5 or 6 . This completes the proof. It has been tacitly assumed that the integral in the right-hand member of (47) is finite; if it is infinite, the theorem is trivial.

HARVARD UNIVERSITY,

Cambridge, Mass. 